\title{
A pure non-gestational ovarian choriocarcinoma with delayed solitary brain metastases: Case report and review of the literature
}

\author{
K. V. L. Narasinga Rao, Subhas Konar, Jagathlal Gangadharan, V. Vikas, S. Sampath \\ Department of Neurosurgery, NIMHANS, Bengaluru, Karnataka, India
}

\begin{abstract}
Choriocarcinoma is the most malignant tumour of gestational trophoblastic origin. Most ovarian choriocarcinomas are gestational in origin and usually metastasize to the ovary from uterine or tubal choriocarcinoma. Non gestational choriocarcinoma (NGOC) of the ovary is exceedingly rare and usually seen along with other germ cell tumors. Non gestational choriocarcinoma has been found to be resistant to single-agent chemotherapy and has a worse prognosis than gestational choriocarcinoma. We are reporting long term follow up of published rare case of pure non gestational ovarian choriocarcinoma (NGOC) with concurrent metastases to the spleen and adrenal glands, who developed a delayed solitary brain metastases, two years after completion of primary treatment. Surgery along with triple agent chemotherapy and radiotherapy was found to give good remission in this aggressive disease.
\end{abstract}

Key words: Delayed metastases, human chorionic gonadotropin, ovarian non-gestational choriocarcinoma

\section{Introduction}

Choriocarcinoma is a rare, highly malignant trophoblastic tumor with gestational or, rarely, germ cell origin. ${ }^{[1]}$ Choriocarcinoma arising in the ovary can have either gestational or non-gestational origin. Gestational ovarian choriocarcinoma may arise in an ovarian pregnancy or represent metastases from intrauterine or intra-tubal primary disease. ${ }^{[2]}$ Non-gestational ovarian choriocarcinoma (NGOC) is exceedingly rare and is usually mixed type associated with other germ cell tumors such as immature teratoma, endodermal sinus tumor, embryonal carcinoma and dysgerminoma. ${ }^{[3]}$ Pure primary NGOC accounts for $<1 \%$ of ovarian tumors. Non-gestational choriocarcinoma of the ovary has a worse prognosis compared to gestational choriocarcinoma. ${ }^{[4]}$ We are reporting long-term follow-up rare case of pure

\begin{tabular}{|l|l|}
\hline \multicolumn{2}{|c|}{ Access this article online } \\
\hline Quick Response Code: & Website: \\
\hline & www.ruralneuropractice.com \\
\cline { 2 - 3 } & \\
\hline & \\
\hline
\end{tabular}

NGOC with concurrent metastases to the spleen and adrenal glands, with delayed solitary brain metastases, 2 years after completion of primary treatment.

\section{Case Report}

A 26-year-old female patient (G1P1) presented with low back ache and vague abdominal pain for 1-month. There were no associated menstrual irregularities or abortions. She was evaluated for these complaints and found to have a right ovarian mass. She was further evaluated by Whole body Fludeoxyglucose positron emission tomography (FDG-PET) scan and CT scan vertex to mid thigh with oral and Intravenous contrast which revealed a lower abdominal mass [Figure 1] with splenic and right adrenal lesions suggestive of metastases [Figure 2]. Increased FDG concentrations were noted in all these lesions. There was no abnormal uptake in the brain or other organs. Her human chorionic gonadotropin (HCG) level was elevated and was $8160 \mathrm{IU} / \mathrm{mL}$. With these findings, she was suspected to have NGOC with ovarian tumor excision (right salpingo-oophorectomy) with partial omentectomy, partial splenectomy and right adrenalectomy in December 2010. Histopathology of the specimen showed pure non-gestational choriocarcinoma.

Address for correspondence:

Dr. K. V. L. Narasinga Rao, Department of Neurosurgery, NIMHANS, Q. No. 27, Type 4, Block-5, Bairasandra Campus, Bengaluru - 560011 , Karnataka, India. E-mail: neuronarsi@gmail.com 
This was, followed by six cycles of chemotherapy with bleomycine, etoposide and vincristine. She was on regular follow-up with HCG levels, which came down to $4.6 \mathrm{IU} / \mathrm{ml}$.

After 2 years of treatment, she presented back to us with a headache, vomitings of 2 months duration and generalized tonic clonic seizures for 10 days. She had papilledema without any motor or sensory deficits. She was evaluated with computed tomogram (CT) of the head that revealed an iso to hyperdense mass lesion with perilesional edema in the left frontal lobe with enhancement on contrast [Figure 3]. There was mass effect and subfalcine herniation. Magnetic resonance imaging of the head revealed a single left superior frontal gyrus solitary mass lesion measuring $3 \mathrm{~cm} \times 3.3 \mathrm{~cm} \times 3.8 \mathrm{~cm}$, which was isointense on T1-Weighted and hyperintense on T2-Weighted images with significant perilesional edema. The lesion was heterogeneously enhancing on contrast. Her serum beta HCG level shot up to $1099 \mathrm{mIU} / \mathrm{ml}$ and cancer antigen - 125 levels was 19-71 IU/ml. Chest X-ray was normal. She underwent left frontal craniotomy and total excision of the lesion. Postoperative CT head revealed complete excision of the lesion. She recovered gradually and was discharged. Histopathology revealed metastatic choriocarcinoma [Figure 4]. She underwent radiotherapy of the brain with $18 \mathrm{~Gy}$ in 10 fractions. Post-radiotherapy the HCG level came down to $12 \mathrm{IU} / \mathrm{mL}$. At 14 months follow-up, patient was asymptomatic with no seizures. Beta HCG level was $<2.0 \mathrm{IU} / \mathrm{mL}$. Chest X-ray and ultrasound abdomen was within normal levels. Follow-up CT scan showed no recurrence of the lesion [Figure 5].

\section{Discussion}

Choriocarcinoma is a malignant gestational trophoblastic disease, which commonly (50\%) occurs after hydatidiform mole. It can occur after a spontaneous abortion in $25 \%$ of cases and after the normal pregnancy in $22.5 \%{ }^{[1,5]}$ Rarely, it may follow ectopic pregnancy and may arise from the ovaries. The tumor is characterized by the presence of two cell lines: The cytotrophoblast, the cells of which lie in sheets to form villus-like structures, and the syncytiotrophoblast, which secretes beta-human chorionic gonadotropin and human placental lactogen and is seen at the advancing edge of the tumor. ${ }^{[6]}$ Most of the primary non-gestational choriocarcinoma of ovary occurs in admixture with teratoma, endodermal sinus tumor, embryonal carcinoma or dysgerminoma ${ }^{[7]}$ Distinction of non-gestational choriocarcinoma from gestational choriocarcinoma is impossible on the histomorphology unless an evidence of pregnancy or other germ cell neoplasm is encountered. DNA polymorphism analysis using two or three appropriate VNTR loci from the tumor and the patient for identification of paternal sequences establishes the diagnosis of gestational or non-gestational choriocarcinoma. ${ }^{[7-8]}$

Ovarian choriocarcinoma with concurrent metastases to spleen and adrenal gland undergoing successful

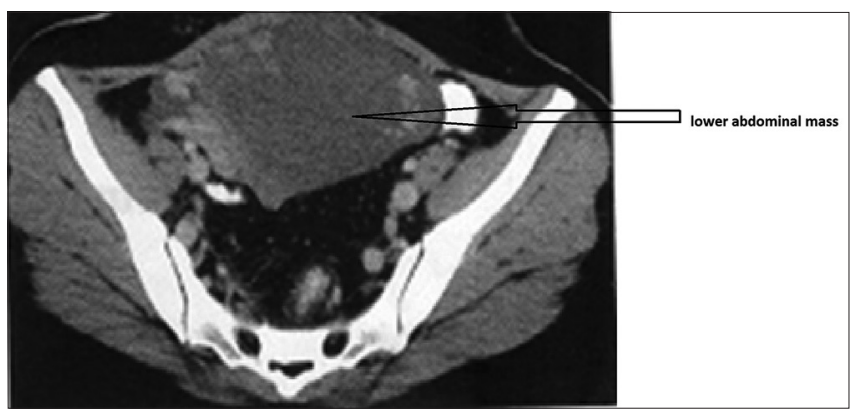

Figure 1: Computed tomogram pelvis with oral and intravenous contrast showing lower abdominal mass as shown by an arrow

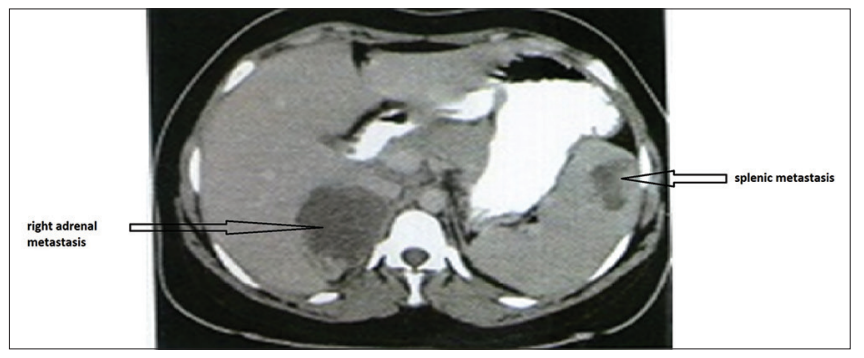

Figure 2: Computed tomogram abdomen with oral and intravenous contrast showing right adrenal and splenic metastases as shown by arrows

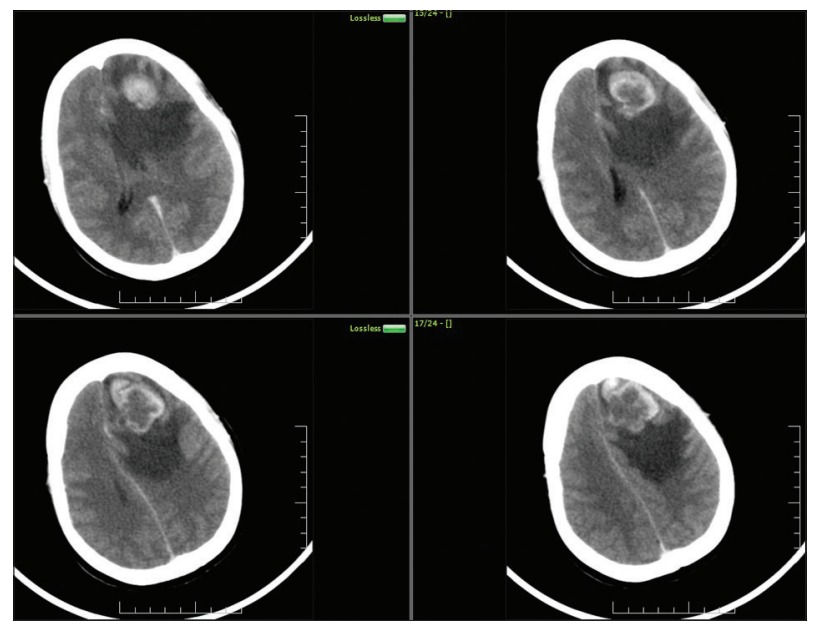

Figure 3: Computed tomogram brain contrast showing single metastasis 


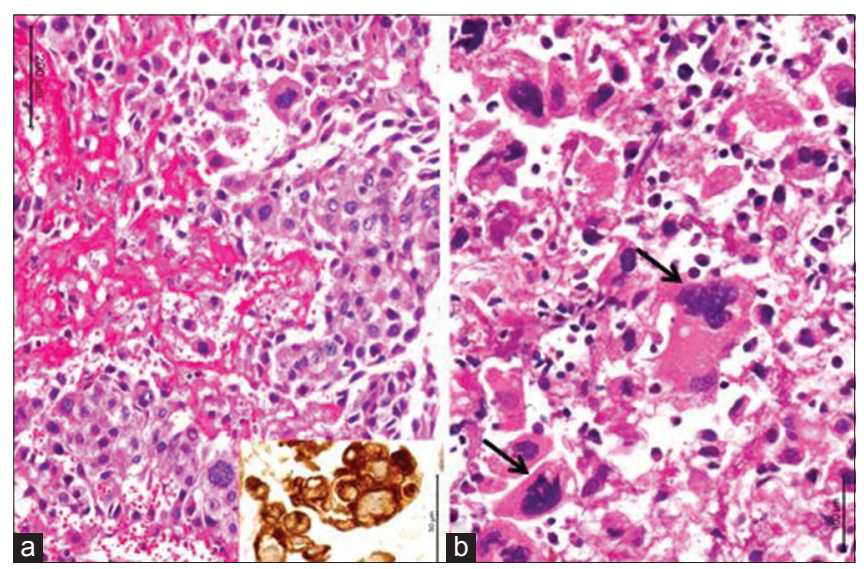

Figure 4: Microphotographs reveal cellular neoplasm with plump tumor cells in broad trabeculae separated by zones of hemorrhage and necrosis (a). The tumors cells are strongly labeled by cytokeratin (a, inset). There are several bizarre tumor giant cells seen (b, arrow). ([a] $\mathrm{H}$ and $\mathrm{E}$, inset: Cytokeratin immunochemistry; [b] $\mathrm{H}$ and $\mathrm{E}$ ) ( magnification $=$ scale bar)

treatment is extremely rare, and this is the only one case, reported in the literature. ${ }^{[5]}$ The tumor has a marked tendency to metastasize early by blood-borne dissemination, to the lungs $(80 \%)$, pelvis $(20 \%)$, vagina (30\%), liver (10\%) and other rare sites including gastrointestinal tract, spleen and kidney. The central nervous system is seldom involved in the absence of pulmonary metastases ${ }^{[6]}$ About $10 \%$ of choriocarcinoma metastases to the brain and is a leading cause of death. ${ }^{[9]}$ Exact incidence of NGOC metastasis to the brain is unknown. The metastasis is commonly hemorrhagic because of the innate capacity of trophoblastic cells to invade and erode vessel walls. ${ }^{[6]}$ In our case, patient presented with concurrent metastases to spleen and adrenal gland and underwent optimal debulking. The patient also received a complete course of chemotherapy. In spite of that, the patient had delayed solitary brain metastases 2 years after completion of chemotherapy, suggestive of aggressive behavior of the tumor.

The treatment of choriocarcinoma consists of polychemotherapy, including regimens that have generally shown to be beneficial with acceptable cure rates and low recurrences. Triple agent chemotherapy is recommended in high risk cases with multiple distant metastases to the liver, lungs or central nervous system, postterm gestational trophoblastic disease and pretreatment serum HCG levels $>40,000 \mathrm{mIU} / \mathrm{ml}$ or interval of $>4$ months since pregnancy. ${ }^{[2,10]}$ Extreme rarity of non-gestational choriocarcinoma hinders therapeutic considerations. However, triple agent chemotherapy has been advocated strongly in these cases, in view of associated poor prognosis. ${ }^{[1,5]}$ Cerebral metastases may

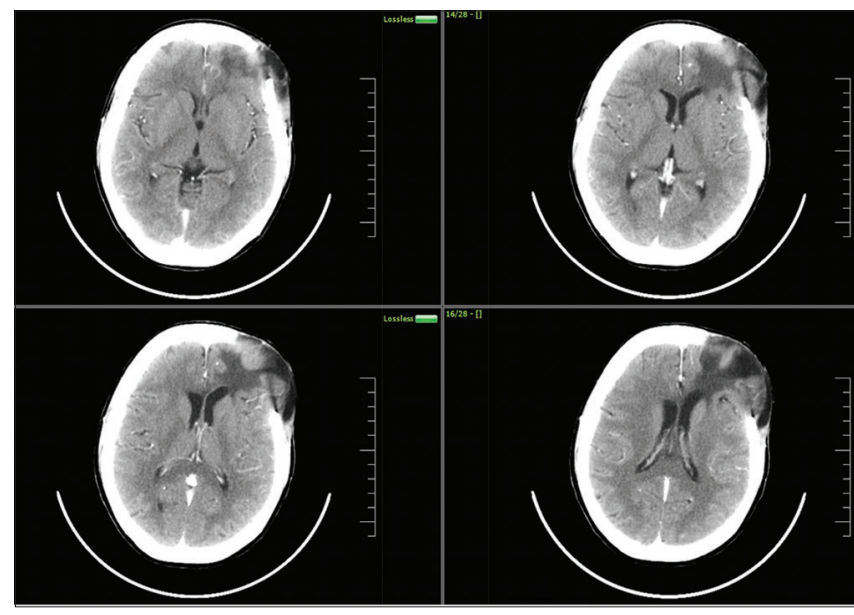

Figure 5: Postoperative computed tomogram contrast at the end of 1 -year showing no recurrence

present while on treatment or as a relapse after partial remission. Metastasis to the brain is considered as a poor prognostic indicator. Beta-HCG is the good predictive indicator of prognosis and recurrence.

\section{Conclusion}

Non-gestational ovarian choriocarcinoma with concurrent metastases to spleen, adrenal is an extremely rare occurrence and is a very aggressive tumor. Despite aggressive management of primary disease, prognosis is poor. Complete excision of the intracranial tumor, followed by chemotherapy and radiotherapy may prolong the survival. A long follow-up with regular beta HCG level estimation is advised to deal with the risk of delayed metastases even when the primary has been well controlled.

\section{References}

1. Ozaki Y, Shindoh N, Sumi Y, Kubota T, Katayama H. Choriocarcinoma of the ovary associated with mucinous cystadenoma. Radiat Med;19:55-9.

2. Axe, Steven R; Klein, Victor R; Woodruff JD. Choriocarcinoma of the Ovary. Obstet and Gynecol 1985;66:111-14.

3. Goswami D, Sharma K, Zutshi V, Tempe A, Nigam S. Nongestational pure ovarian choriocarcinoma with contralateral teratoma. [Internet]. Gynecol Oncol 2001;80:262-6.

4. Scully RE. Tumors of the ovary and mal-developed gonads. In: Hartmann WH, editor. Atlas of tumor pathology. Washington, DC: Armed Forces Institute of Pathology; 1979 p. 243-5.

5. Ramarajapalli ML, Rao NAR, Murudaraju P, Kilara NG. Ovarian Choriocarcinoma with Concurrent Metastases to the Spleen and Adrenal Glands: First Case Report. J Gynecol Surg 2012;28:153-155.

6. Kidd D, Plant GT, Scaravilli F, McCartney ACE, Stanford M, Graham EM. Metastatic choriocarcinoma presenting as multiple intracerebral haemorrhages: The role of imaging in the elucidation of the pathology. J Neurol Neurosurg Psychiatry 1998;65:939-41.

7. Tsujioka H, Hamada H, Miyakawa T, Hachisuga T, Kawarabayashi T. A pure nongestational choriocarcinoma of the ovary diagnosed with DNA polymorphism analysis. Gynecol Oncol 2003 Jun;89:540-542. 
Rao, et al.: Pure non-gestational ovarian choriocarcinoma metastasis to brain

8. Koo H-L, Choi J, Kim K-R, Kim J-H. Pure non-gestational choriocarcinoma of the ovary diagnosed by DNA polymorphism analysis. Pathol Int 2006;56:613-6.

9. Dadlani R, Furtado S, Ghosal N, Prasanna K, Hegde AS. Unusual clinical and radiological presentation of metastatic choriocarcinoma to the brain and long-term remission following emergency craniotomy and adjuvant EMA-CO chemotherapy. J Cancer Res Ther;6:552-6.
10. Berkowitz RS, Goldstein DP. Chorionic tumors. The N Engl J Med 1996;335:1740-8.

How to cite this article: Rao KN, Konar S, Gangadharan J, Vikas V, Sampath S. A pure non-gestational ovarian choriocarcinoma with delayed solitary brain metastases: Case report and review of the literature. J Neurosci Rural Pract 2015;6:578-80.

Source of Support: Nil. Conflict of Interest: None declared. 